\title{
Olanzapine use in a manic patient during second and third trimester pregnancy
}

\author{
This article was published in the following Dove Press journal: \\ Neuropsychiatric Disease and Treatment \\ 17 February 2014 \\ Number of times this article has been viewed
}

\author{
Lynn Choi \\ Soo-Hyun Joo \\ Jong-Hyun Jeong \\ Department of Psychiatry, St Vincent's \\ Hospital, College of Medicine, \\ The Catholic University of Korea, \\ Suwon, Korea
}

\begin{abstract}
Women with bipolar disorder have a high risk for symptom exacerbation during pregnancy and the risk is elevated further when mood stabilizers are discontinued. This report describes a 31-year-old bipolar woman who discontinued medication before pregnancy but had to resume her pharmacotherapy due to manic episodes that recurred during the second trimester. Olanzapine, an atypical antipsychotic, was administered from week 25 of gestation and then replaced with quetiapine in week 35 of gestation. Even though a consensus on clinical interventions for pregnant patients with symptom relapse has not been reached, clinicians should still discuss pregnancy and therapeutic management with every female bipolar patient of childbearing age. This discussion is important because treatment can be managed most effectively in these individuals if pregnancy is planned. Ultimately, clinical decisions should be made on a case-by-case basis, weighing the risks to the mother and fetus between the disorder itself and the teratogenicity of pharmacotherapy.
\end{abstract}

Keywords: pregnancy, bipolar disorder, olanzapine, manic

\section{Introduction}

During pregnancy and the postpartum period, women with psychiatric disorders are at high risk for relapse and symptom exacerbation, and even show de novo emergence of psychiatric disorders. In the case of bipolar disorder, pregnancy has historically been regarded as a protective factor and to improve the clinical course of the illness. ${ }^{1}$ However, recent studies have shown that relapse rates for bipolar disorder during pregnancy can reach $50 \%$, casting doubt on the role of pregnancy as a protective factor. ${ }^{2-6} \mathrm{~A}$ recent prospective study found that $70.7 \%$ of patients with bipolar I or II disorder underwent at least one mood-destabilizing episode during pregnancy, and the risk of relapse was higher for those who stopped taking their medication. ${ }^{7}$ Even with a high risk of recurrence without treatment, only $5.5 \%$ of patients receive appropriate pharmacotherapy, ${ }^{8}$ which is most likely to be due to incomplete safety data for pregnant patients and the known teratogenicity of agents frequently employed to manage the illness. Herein, we describe a pregnant patient with bipolar disorder and discuss clinical issues regarding her psychopharmacotherapy.

\section{Case report}

A 31-year-old woman with a history of four psychiatric hospitalizations (for a depressive episode in 2002 and three manic episodes thereafter) visited our clinic in 2010 and began a course of valproic acid $500 \mathrm{mg} /$ day and olanzapine $5 \mathrm{mg}$ /day. She demonstrated stable progress and was in a euthymic state while discussing pregnancy. 
The clinical staff planned to taper out the mood stabilizer slowly, use only quetiapine and clonazepam just prior to pregnancy, and cease all medication by June 1, 2012. After one month, her pregnancy was confirmed.

In September 2012, the patient experienced nausea and vomiting and was admitted to the obstetric department for conservative care. She then complained of depressed mood, avolition, and loss of appetite in weeks 9-10 of gestation. After some time, the patient developed a manic episode with elated mood, decreased need for sleep, talkativeness, hyperactivity, and distractibility, at which time she was admitted to the inpatient psychiatric department (January 2013).

After hospitalization, the clinical team decided to control the patient's symptoms by close monitoring without pharmacotherapy. However, due to her persistent irritability and violent behavior, it was decided that overall harm to the fetus from the disorder exceeded that from pharmacotherapy. Electroconvulsive therapy, which the clinical team considered as the first-line treatment, was not carried out due to her family's refusal. Therefore, a course of olanzapine, which was shown to be effective in previous years, was reinstituted at week 25 of gestation with a dose of up to $7.5 \mathrm{mg} /$ day. However, she was discharged early, even with symptom persistence, due to the family's demands. Despite using a $7.5 \mathrm{mg}$ /day dose of olanzapine, she was readmitted in March 2013 (week 33 of gestation) for persecutory delusions and delusions of infidelity in addition to manic symptoms.

Despite amelioration of the delusions after taking olanzapine up to $20 \mathrm{mg} /$ day, the patient still continued to show irritability and behavioral disturbances. Therefore, during week 35 of gestation, her medication was switched from olanzapine to quetiapine. Her sleep duration lengthened and she showed some improvements in irritability and hyperactivity afterwards with $800 \mathrm{mg}$ /day of quetiapine, but still appeared to be talkative and sometimes temperamental. She was found to have oligohydramnios on follow-up sonogram, and emergency cesarean section was carried out at week 37 of gestation. The patient experienced no postsurgical complications, and the newborn's Apgar score was 9. The baby was deemed to be healthy by the pediatrician and there were no congenital malformations.

Beginning the day after surgery, the patient was started on valproic acid 1,000 mg/day and quetiapine $800 \mathrm{mg} /$ day and showed improvement in her irritability, loss of impulse control, and distractibility. After agreeing not to breastfeed, the valproic acid was increased to $1,500 \mathrm{mg} /$ day based on blood levels of $44.53 \mu \mathrm{g} / \mathrm{mL}$ while taking $1,000 \mathrm{mg} /$ day. She tolerated the medication even after quetiapine was increased to_1,200 mg/day; she showed stable progress and was discharged on the 59th inpatient day.

\section{Discussion}

The present case describes a bipolar patient who ceased pharmacotherapy ahead of pregnancy but had to resume medication in her second trimester due to recurrence of manic episodes. A recent prospective study found a higher risk of recurrence of mood episodes for patients who discontinued mood stabilizers (85.5\%), as compared with those still taking mood stabilizers $(37 \%),{ }^{7}$ and the risk of recurrence was even higher for those who discontinued medication abruptly. Other predictors of recurrence of mood episodes following discontinuation of mood stabilizers include a diagnosis of bipolar II disorder, early onset, unplanned pregnancy, more recurrences per year, recent illness, use of antidepressants, and use of anticonvulsants instead of lithium. ${ }^{7}$ Some investigators have concluded that biological factors such as younger age, onset of illness at an early age, prenatal mood episodes, and obstetric/somatic complications during pregnancy were associated with childbearing-related episodes. ${ }^{3}$ In this case, the patient had a thorough discussion about pregnancy with her clinician, tapered off her medication slowly ahead of conception, and showed stable progress. However, she developed unexpected depressive and manic episodes at 3 and 5 months' gestation.

Clinicians must weigh the relative risks of medications administered during pregnancy and the associated risk of relapse if pharmacologic treatment is discontinued. For women who are required or prefer to continue pharmacotherapy during the first trimester, the lowest effective dose of a medication must be used, and agents with the lowest teratogenic potential should be selected.

The US Food and Drug Administration classifies drug use during pregnancy into five categories to address and inform clinicians about the risks of fetal exposure, and this classification is currently being revised. Lithium, a gold standard treatment for bipolar disorder, was classified as a category D medication, as were valproate and carbamazepine. Historically, it was recommended that patients cease lithium during pregnancy because first-trimester exposure yielded greater rates of cardiovascular malformation compared with that in the general population. However, recent studies have shown the teratogenic risk to be lower than originally believed and advise continuation of lithium given the effectiveness of this pharmacotherapy for mood episodes. ${ }^{9}$ Because exposure to valproate 17-30 days after conception is associated with a $5 \%-9 \%$ risk of neural tube defects, it is 
generally recommended that valproate be switched to another mood stabilizer before conception and in early pregnancy.

The American Congress of Obstetricians and Gynecologists recommends continuing pharmacotherapy during pregnancy because severe psychiatric episodes are generally thought to be caused by discontinuation of medication. ${ }^{10-12}$ Some experts consider the risk associated with firstgeneration antipsychotic agents, which have been available for decades, to be less than the risk associated with selected mood stabilizers. ${ }^{13,14}$ However, in the case of atypical antipsychotics, it is difficult to draw any definitive conclusions due to a paucity of reproductive safety data, ${ }^{15}$ so routine use is not recommended during pregnancy.

Olanzapine, which was administered in this case, was not found to be associated with an increased risk of major malformations even when administered at 9-30 times the maximum dosage in animal studies. However, it was found to be associated with low birth weight in a dose-dependent manner. Outcomes from 23 prospectively ascertained olanzapine-exposed pregnancies showed a $13 \%$ rate of spontaneous abortion, $5 \%$ stillbirth, $0 \%$ major malformations, and $5 \%$ prematurity, all within the range of normal historic control rates. ${ }^{16}$ Another study of 18 pregnancies yielded similar results, suggesting that olanzapine is relatively safe when used in pregnancy. ${ }^{17}$

Quetiapine was not found to be associated with an increased risk of major malformations, but some minor malformations in the upper and lower limbs in animal studies and low birth weight were observed when used in higher dosages. ${ }^{18}$ In the case of human exposure, there are a number of case reports of quetiapine-exposed pregnancies that did not produce any malformations, ${ }^{19-21}$ even though there has not been a systematic report developed. In addition, some investigators have reported that quetiapine shows the lowest placental passage when compared with olanzapine, haloperidol, and risperidone. ${ }^{22}$

When reviewing studies and case reports to date, one might discern that typical and atypical antipsychotics have a better safety record when used during pregnancy than conventional mood stabilizers. However, in practice, treatment decisions need to be made by balancing the risks and benefits of various treatment strategies, taking into consideration the needs of the individual patient.

In summary, optimal treatment planning for women with bipolar disorder emphasizes overall care to promote both preconception and prenatal health. Treatment planning is critical for minimizing the risk to the mother and fetus while limiting the morbidity from active psychiatric illness. In order to assist their patients in making the best choices for the health of the mother and fetus, clinicians must remain familiar with the latest productive safety research of any medications used to treat the disorder.

\section{Disclosure}

The authors reports no conflict of interest in this work.

\section{References}

1. Grof P, Robbins W, Alda M, et al. Protective effect of pregnancy in women with lithium-responsive bipolar disorder. J Affect Disord. 2000;61(1-2):31-39.

2. Freeman MP, Smith KW, Freeman SA, et al. The impact of reproductive events on the course of bipolar disorder in women. J Clin Psychiatry. 2002;63(4):284-287.

3. Akdeniz F, Vahip S, Pirildar S, Vahip I, Doganer I, Bulut I. Risk factors associated with childbearing-related episodes in women with bipolar disorder. Psychopathology. 2003;36(5):234-238.

4. Jones I, Craddock N. Bipolar disorder and childbirth: the importance of recognising risk. Br J Psychiatry. 2005;186:453-454.

5. Sharma V, Pope CJ. Pregnancy and bipolar disorder: a systematic review. J Clin Psychiatry. 2012;73(11):1447-1455.

6. Bodén R, Lundgren M, Brandt L, Reutfors J, Andersen M, Kieler H. Risks of adverse pregnancy and birth outcomes in women treated or not treated with mood stabilisers for bipolar disorder: population based cohort study. BMJ. 2012;345:e7085.

7. Viguera AC, Whitfield T, Baldessarini RJ, et al. Risk of recurrence in women with bipolar disorder during pregnancy: prospective study of mood stabilizer discontinuation. Am J Psychiatry. 2007;164(12): 1817-1824.

8. Andersson L, Sundström-Poromaa I, Bixo M, Wulff M, Bondestam K, åStröm M. Point prevalence of psychiatric disorders during the second trimester of pregnancy: a population-based study. Am J Obstet Gynecol. 2003;189(1):148-154.

9. Jacobson SJ, Jones K, Johnson K, et al. Prospective multicentre study of pregnancy outcome after lithium exposure during first trimester. Lancet. 1992;339(8792):530-533.

10. Viguera AC, Cohen LS, Baldessarini RJ, Nonacs R. Managing bipolar disorder during pregnancy: weighing the risks and benefits. Can J Psychiatry. 2002;47(5):426-436.

11. Yonkers KA, Wisner KL, Stowe Z, et al. Management of bipolar disorder during pregnancy and the postpartum period. Am J Psychiatry. 2004;161(4):608-620.

12. ACOG Committee on Practice Bulletins - Obstetrics. ACOG Practice Bulletin: Clinical management guidelines for obstetrician-gynecologists number 92, Apr 2008 (replaces practice bulletin number 87, Nov 2007). Use of psychiatric medications during pregnancy and lactation. Obstet Gynecol. 2008;111(4):1001-1020.

13. McKenna K, Koren G, Tetelbaum M, et al. Pregnancy outcome of women using atypical antipsychotic drugs: a prospective comparative study. J Clin Psychiatry. 2005;66(4):444-449.

14. Yaeger D, Smith HG, Altshuler LL. Atypical antipsychotics in the treatment of schizophrenia during pregnancy and the postpartum. Am J Psychiatry. 2006;163(12):2064-2070.

15. Cohen LS. Treatment of bipolar disorder during pregnancy. J Clin Psychiatry. 2007;68 Suppl 9:4-9.

16. Goldstein DJ, Corbin LA, Fung MC. Olanzapine-exposed pregnancies and lactation: early experience. J Clin Psychopharmacol. 2000;20(4):399-403.

17. Biswasl PN, Wilton LV, Pearcel GL, Freemantle S, Shakir SA. The pharmacovigilance of olanzapine: results of a post-marketing surveillance study on 8858 patients in England. J Psychopharmacol. 2001;15(4):265-271. 
18. Seroquel product information. Wilmington, DE, USA: AstraZeneca Pharmaceuticals; 2003.

19. Tényi T, Trixler M, Keresztes Z. Quetiapine and pregnancy. Am $J$ Psychiatry. 2002;159(4):674.

20. Gentile S. Quetiapine-fluvoxamine combination during pregnancy and while breastfeeding. Arch Womens Ment Health. 2006;9(3): $158-159$.
21. Frayne J, Nguyen T, Kohan R, De Felice N, Rampono J. The comprehensive management of pregnant women with major mood disorders: a case study involving phenelzine, lithium, and quetiapine. Arch Womens Ment Health. November 8, 2013. [Epub ahead of print.]

22. Newport DJ, Calamaras MR, DeVane CL, et al. Atypical antipsychotic administration during late pregnancy: placental passage and obstetrical outcomes. Am J Psychiatry. 2007;164(8):1214-1220.

\section{Publish your work in this journal}

Neuropsychiatric Disease and Treatment is an international, peerreviewed journal of clinical therapeutics and pharmacology focusing on concise rapid reporting of clinical or pre-clinical studies on a range of neuropsychiatric and neurological disorders. This journal is indexed on PubMed Central, the 'PsycINFO' database and CAS.
The manuscript management system is completely online and includes a very quick and fair peer-review system, which is all easy to use. Visit http://www.dovepress.com/testimonials.php to read real quotes from published authors.

\footnotetext{
Submit your manuscript here: http://www.dovepress.com/neuropsychiatric-disease-and-treatment-journal
} 